\title{
Biologic Agent-Associated Cutaneous Adverse Events: A Single Center Experience
}

\author{
Rahul Sehgal, MD; Erik J. Stratman, MD; and Jonathan E. Cutlan, MD \\ Biologic agents are regarded as an effective treatment for a variety of autoimmune diseases. These \\ drugs have an acceptable safety and tolerability profile, although an increasing number of autoimmune \\ conditions have been reported with their use. Additionally, a variety of cutaneous diseases have been \\ associated with their use. Here we report our experience of adverse cutaneous events with the use \\ of biologic agents. An alternative explanation for patients presenting with adverse cutaneous events \\ including drug interactions must be carefully investigated.
}

Keywords: Adverse drug events; Anti-TNF therapy; Autoimmune disease; Biologic agents; Cutaneous vasculitis

$\mathrm{B}$ iologic agents (aka. biologics) are geneticallyengineered proteins derived from human genes designed to reduce inflammation by inhibiting specific components of the immune system that can cause inflammation. Biologics are used in treating a wide variety of autoimmune diseases and have become mainstays in the management of rheumatoid arthritis (RA), ankylosing spondylitis, psoriasis, psoriatic arthritis (PsA), and inflammatory bowel disease (IBD). These agents have an acceptable safety and tolerability profile, although an increasing number of autoimmune conditions related to their use have been reported. ${ }^{1}$ A variety of cutaneous diseases are also reported as being induced by biologic agents. ${ }^{2}$ In a large prospective study of patients with RA, $25 \%$ of those on tumor necrosis factor- $\alpha$ inhibitor (TNFI) therapy required a dermatological consultation, compared with $13 \%$ among a TNF-I naïve group. ${ }^{3}$ Skin infections, eczema, and drug-related eruptions are the most common cutaneous adverse events reported. Immune-mediated skin lesions attributed to use of biologic agents include psoriasis, granuloma annulare, cutaneous vasculitis, alopecia areata, cutaneous lupus erythematosus, lichen planus, hidradenitis suppurative, cutaneous sarcoidosis, and vitiligo. ${ }^{4}$ Psoriasis is the most often reported of these immune mediated skin lesions. $^{5}$
We report, herein, our experience with cutaneous adverse events associated with the use of biologic agents.

\section{Case I}

A man, aged 58 years, presented with a 6-month history of an acute onset bright red skin rash. His chronic psoriasis and PsA had been under control with etanercept monotherapy for the past 15 years. The newer eruption consisted of a partially blanchable, confluent dusky red patch extending over $30 \mathrm{~cm}$, from just below the umbilicus to the superior abdomen with extension on to the lateral abdomen, groin, and scrotum (Figure 1, panels A and B). His serologic profile was negative for rheumatoid factor $(\mathrm{RF})$ and anti-cyclic citrullinated peptide antibody (ACPA). Antinuclear antibody (ANA) was weakly positive at a titer of 80 . Erythrocyte sedimentation rate (ESR) was $27 \mathrm{~mm} / \mathrm{h}$ (range $0-13 \mathrm{~mm} / \mathrm{h}$ ); C-reactive protein (CRP) was $0.2 \mathrm{mg} / \mathrm{dL}$ (range $\leq 1.0 \mathrm{mg} / \mathrm{dL}$ ). The patient underwent two punch biopsies showing a moderately dense superficial to deep perivascular and interstitial, predominantly neutrophilic infiltrate with occasional eosinophils, no vasculitis, and no alteration of dermal collagen (Figure 2, panels A and B), consistent with a neutrophilic dermatosis (ND). Aerobic, fungal, and mycobacterial tissue cultures were negative. A series of treatments were attempted, including oral prednisone,

Submitted: March 16, 2017

Revised: November 12, 2017

Accepted: December 15, 2017
Corresponding Author: Rahul Sehgal, MD, Mayo Clinic Health System-Eau Claire, Department of Rheumatology, I 400 Bellinger Street, Eau Claire, WI 54702 USA, Email: Sehgal.rahul@mayo.edu 
dapsone, and colchicine without resolution of the eruption. These treatments and etanercept were then discontinued. The patient was started on treatment with adalimumab for worsening psoriatic arthritis with good clinical response. His skin condition completely resolved within 8 weeks without flare-up of psoriatic arthritis or psoriasis. No recurrence of skin rash has been observed over the subsequent year (Figure 1, panels $\mathrm{C}$ and $\mathrm{D})$.

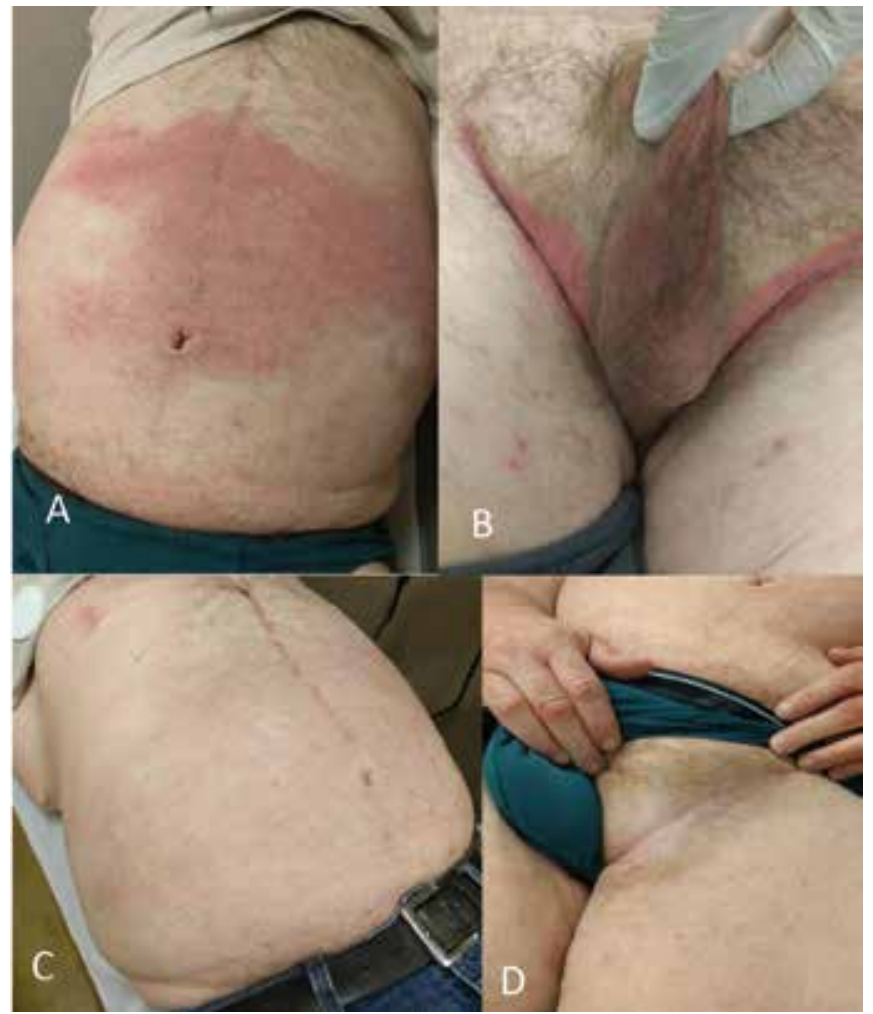

Figure 1. (A, B) Cutaneous rash while on etanercept. (C, D) 8 weeks after discontinuation of etanercept and initiation of adalimumab the rash has disappeared.

\section{Case 2}

A woman, aged 65 years, with a $>30$ year history of nodular and erosive RA presented with a new rash. She had a history of active RA with a routine assessment of patient index data 3 score of 24 . Her serologic profile was strongly positive for RF at a titer of 1:1280 (nL $<1: 80)$, ACPA $>300(\mathrm{~nL}<2.9)$, ANA 1:80, ESR $67 \mathrm{~mm} / \mathrm{h}$, and CRP $4 \mathrm{mg} / \mathrm{dL}$. In view of her persistent RA activity despite previous methotrexate and hydroxychloroquine therapy, certolizumab pegol injection was initiated. Within 20 hours of initiating treatment, nonblanching, violaceous-red pinpoint to $0.3 \mathrm{~cm}$ petechial and purpuric macules appeared on dorsal feet extending up to shins/calves, thighs, medial buttocks, and supra-pubic area (Figure 3, panels A and B). Biopsy of the left medial dorsal foot showed prominent extravasated red blood cells with associated neutrophils, leukocytoclasis, and perivascular deposition of immunoglobulin [IG]-G, IG-M, complement-3, and fibrinogen on direct immunofluorescence (DIF) consistent with leukocytoclastic vasculitis (Figure 4). Her renal function remained normal (creatinine $0.9 \mathrm{mg} / \mathrm{dL}$ ). Certolizumab was discontinued, and the skin symptoms resolved over the next 10 days. The patient has since been managed with combination treatment comprised of methotrexate, hydroxychloroquine, and low dose leflunomide, which has maintained adequate control of her RA without recurrence of cutaneous lesions. Interestingly, a previous trial of adalimumab 3 years prior had resulted in acute sarcoidosis with shortness of breath, new onset hilar/mediastinal lymphadenopathy, and progressive, confluent, non-blanchable, macules, petechial, and purpuric eruption on the abdomen extending onto the pelvis, lower back, buttock, feet, and lower legs. A mediastinal lymph node biopsy (Figure 5, panels A and B) confirmed non-caseating granuloma consistent with sarcoidosis. An evolving perivascular granulomatous inflammation was noted on a skin biopsy from her leg. Adalimumab was thereafter discontinued with gradual resolution of skin lesions.

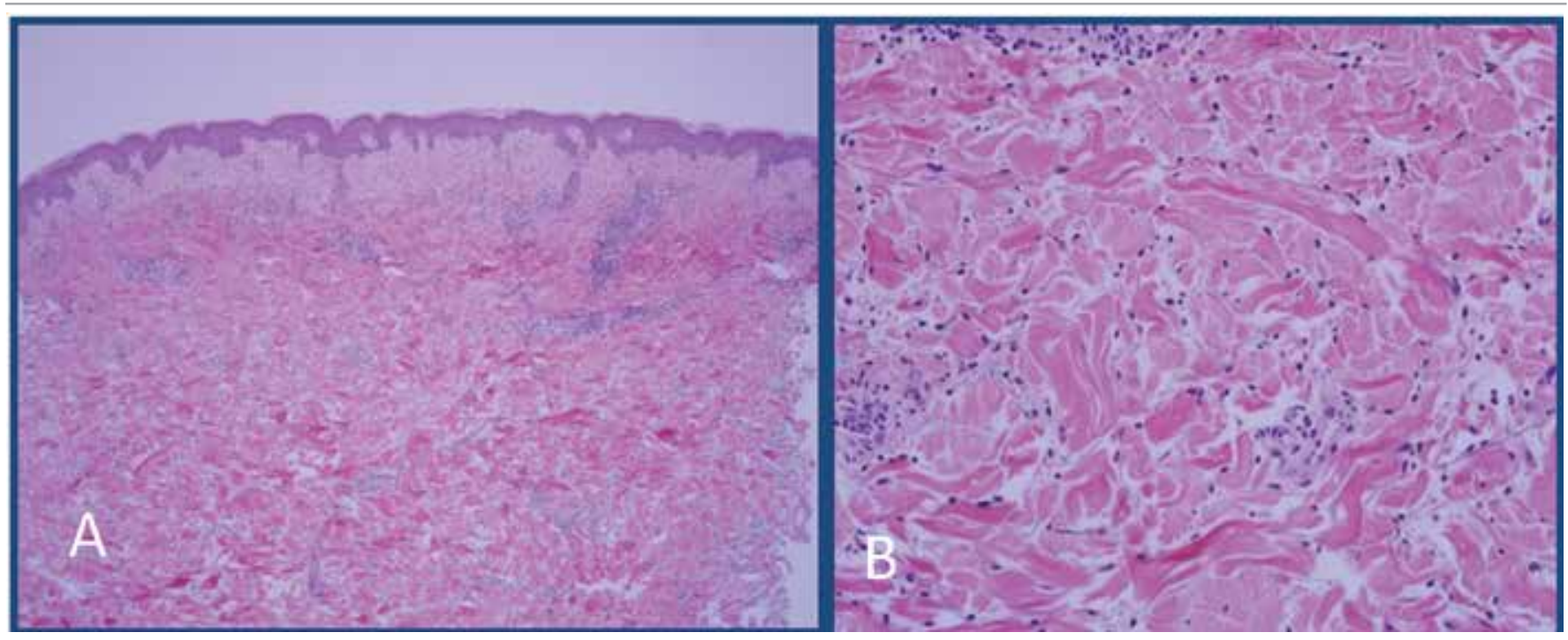

Figure 2. (A) Biopsy of the left lateral abdomen shows mild superficial dermal edema and interstitial and perivascular inflammation (10X, hematoxylin and eosin). (B) High power image showing that the majority of the inflammatory cells are interstitial and perivascular neutrophils. The blood vessels fail to show vascular damage (20X, hematoxylin and eosin). 


\section{Case 3}

A woman, aged 29 years, developed numerous red and scaly, round to oval plaques over her back, breasts, inframammary and infrapannus regions, and legs (Figure 6, panels A, B, C) after 3 weeks of receiving tocilizumab treatment for Takayasu arteritis. She was diagnosed 6 years earlier in the setting of resistant hypertension, absent left forearm pulse, and marked elevation of acute phase reactants (ESR $85 \mathrm{~mm} / \mathrm{h}$; C-reactive protein $11 \mathrm{mg} / \mathrm{dL}$ ). She had extensive disease and was found to have external carotid, renal, subclavian, celiac, and vertebral artery inflammation and obstruction. She was previously treated with methotrexate, azathioprine, infliximab, rituximab and high dose systemic corticosteroids. Tocilizumab was initiated as a steroid sparing alternative. The skin lesions worsened after her second infusion of tocilizumab. Skin biopsy showed features of psoriasiform epidermal hyperplasia, confluent parakeratosis, loss of granular layer and dilated blood vessels within dermal papillae consistent with a diagnosis of psoriasis (Figure 7). Tocilizumab treatment was discontinued. Psoriasis was treated with topical steroids and narrow band ultraviolet B therapy with improvement. Ustekinumab was started for vasculitis progression, resulting in resolution of skin psoriasis and vasculitis.

\section{Discussion}

The cases we describe include two unique patients on TNF-I therapy who developed unusual drug-associated adverse cutaneous eruptions including ND associated with etanercept therapy, cutaneous vasculitis associated with use of certolizumab therapy, and psoriasis occurring in a patient with Takayasu arteritis treated with tocilizumab. A variety of immune mediated reactions and other adverse events have been reported with use of biologic agents. Cutaneous manifestations are most common among these adverse events. In majority $(80 \%)$ of cases, these adverse events represent a new disease while in some $(20 \%)$ they are exacerbations of pre-existing disease. ${ }^{6}$ In a prospective study,

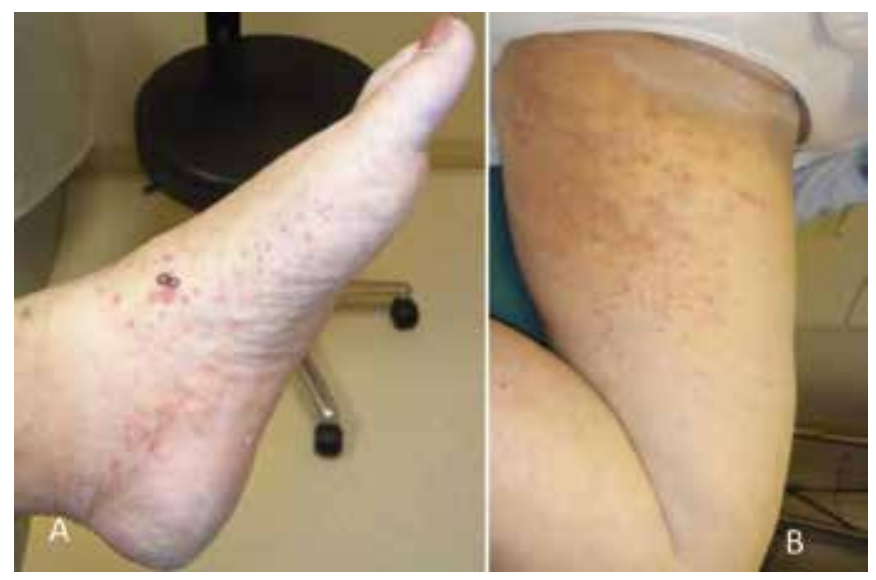

Figure 3. Figure showing extent and distribution of cutaneous vasculitis lesions on (A) the foot and (B) inner thigh of the patient on certolizumab.

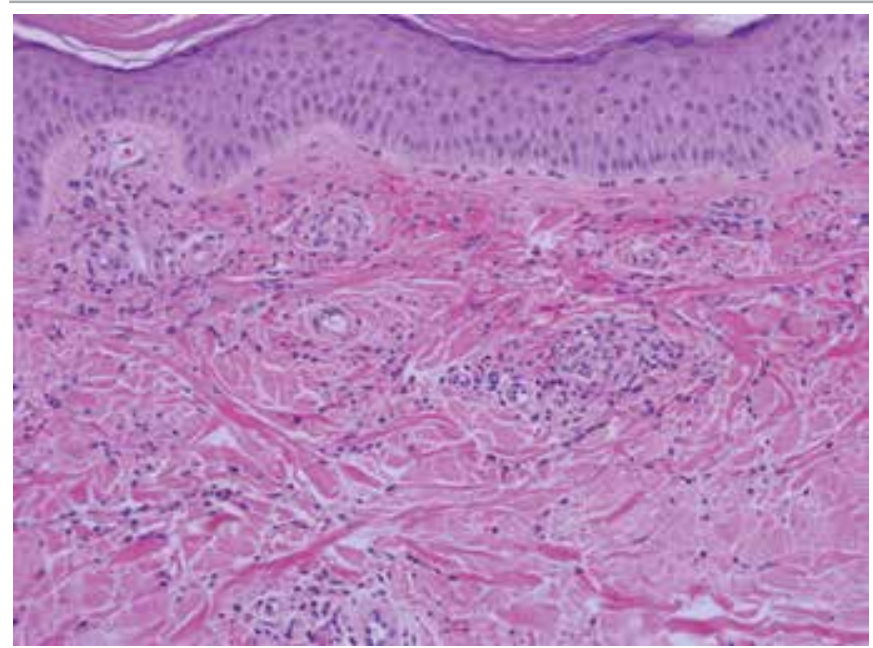

Figure 4. Biopsy of the left medial dorsal foot shows prominent extravasated red blood cells with associated neutrophils with leukocytoclasis and perivascular fibrin deposition, consistent with a leukocytoclastic vasculitis (20X, hematoxylin and eosin).

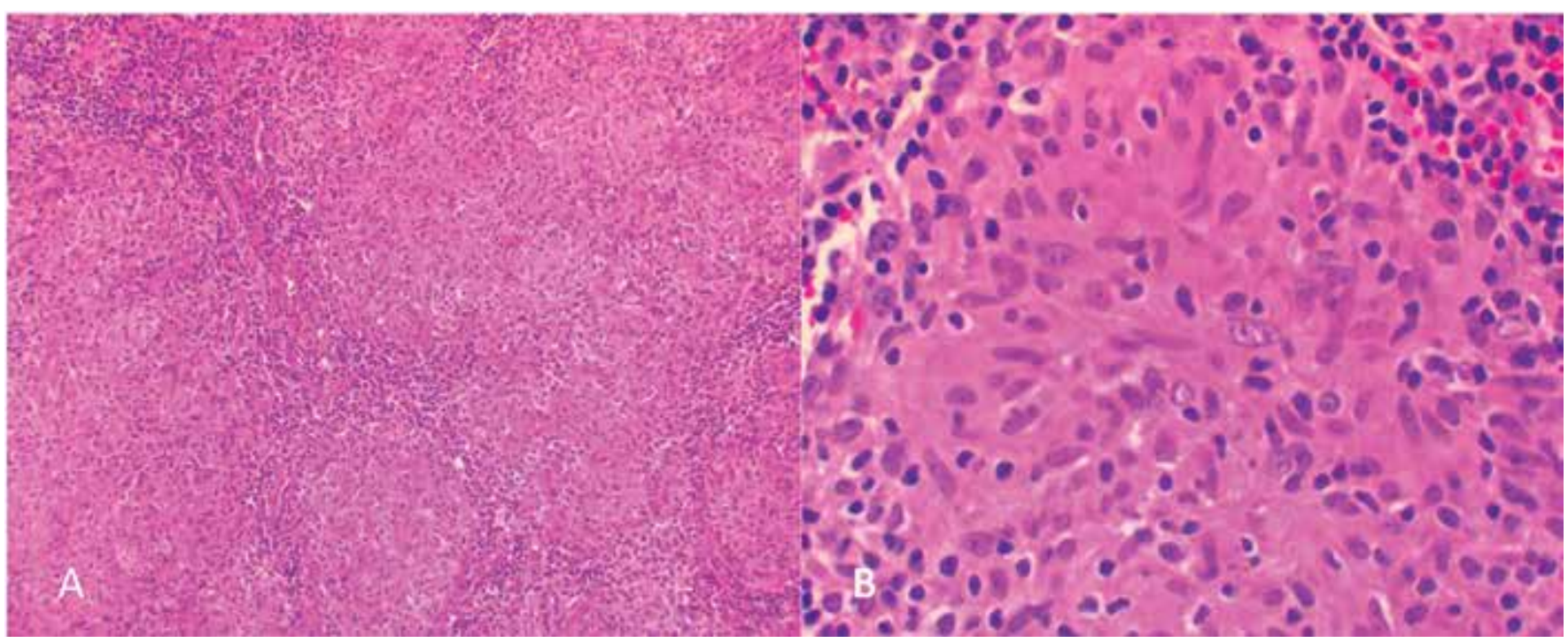

Figure 5. (A, B) Sections of the mediastinal lymph node show diffuse non-necrotizing granulomatous inflammation, consistent with sarcoidosis. Special stains [(A) GMS and (B) FITE] were negative for micro-organisms. 
Table 1. Medications associated with neutrophilic dermatoses ${ }^{12}$

All-transretinoic acid
Carbamazepine
Celecoxib
Diazepam
Diclofenac
Granulocyte colony-stimulating factor
Levonorgestrel / ethinyl estradiol
Hydralazine
Minocycline
Nitrofurantoin
Propylthiouracil
Trimethoprim-sulfamethoxazole

dermatological events resulted in withdrawal of TNF-I therapy in $26 \%$ of patients with RA. ${ }^{3}$ Other than infusion/injection site reactions, psoriasis and psoriasiform-like lesions, lupus-like syndromes, cutaneous vasculitis, and cutaneous infections have the strongest association with TNF-I treatment. These are followed by eczematous reactions, lichenoid disorders, and granulomatous reactions. There has not been an association with use of TNF-I treatment and cutaneous lymphoma, epithelial skin cancers or melanoma. ${ }^{7}$ Cutaneous reactions such as infusion reactions and injection site reactions can be directly attributed to the administration of TNF-I therapy. However, a direct association with use of TNF-I and other cutaneous reactions is not always obvious.

The patient reported in case 1 developed ND while on etanercept therapy. Neutrophilic dermatoses (ND) are a group of diseases including Sweet syndrome (SS), pyoderma gangrenosum (PG), subcorneal pustular dermatosis (SPD), and erythema elevatum diutinum. ${ }^{8}$ As the name implies, ND are characterized by a dense inflammatory infiltrate composed of neutrophils in absence of infection. A number of systemic disorders such as RA, systemic lupus erythematosus, dermatomyositis, systemic sclerosis, IBD, myeloproliferative disorders, monoclonal gammopathy and auto-inflammatory diseases are associated with development of ND. A wide variety of drugs ${ }^{9}$ have also been implicated with development of SS. Granulocyte colony stimulating factor (G-CSF) is considered most common among them while other drugs include minocycline, trimethoprim/sulfamethoxazole, HRT/ OCP, furosemide, celecoxib, azathioprine..$^{10}$ TNF-I have also been associated with development of $\mathrm{SS}^{11,2}$ and $\mathrm{PG}$ lesions. ${ }^{12}$ Similar unexpected reactions have been described with infliximab use in $\mathrm{UC}^{13}$ and $\mathrm{RA}^{14}$ (Table 1).

The occurrence of ND in our patient on etanercept treatment cannot be attributed to class effect of TNF-I therapy, since adalimumab, etanercept and infliximab have been used successfully in managing refractory PG associated with IBD. ${ }^{15,16}$ Neutrophilic dermatosis (ND) and especially SS have also been successfully treated with TNF-I such as etanercept and infliximab. ${ }^{17}$ Success with use of TNF-I has been rather variable in SPD. The treatment achieved complete remission in one case $^{18}$ while the results were temporary in another case. $^{19}$

A lack of response to systemic treatment in our case 1 and skin improvement upon etanercept discontinuation with a switch to adalimumab favors its association with etanercept use. The pathophysiology of ND is poorly understood. An underlying role of activated $\mathrm{T}$ lymphocyte in recruitment of neutrophils to the dermis has been postulated.

Cytokine expression in skin of PG and SS patients compared with healthy controls show an increased expression of IL-1, IL-8, IL-17, TNF-alpha, MMP-2, MMP-9, IFN- $\gamma$, and $\mathrm{G}-\mathrm{CSF}^{20}$

Cutaneous vasculitis as described in our case 2 has been reported with TNF-I therapy (especially etanercept and infliximab) in RA patients. ${ }^{21,22}$ Although a direct causal relationship of drug use with cutaneous vasculitis is difficult to establish, a close temporal association of vasculitis with initiation of TNF-I therapy and its resolution upon discontinuation of the drug aids in this distinction. The pathogenesis of cutaneous vasculitis occurrence is hypothesized to be a type 3 hypersensitivity reaction triggered by anti-TNF- $\alpha$ :TNF- $\alpha$ immune complex deposition in small capillaries and the induction of complement activation. ${ }^{23}$ Management of cutaneous vasculitis in majority of affected patients involves discontinuation of the offending drug, in this case the TNF-I therapy, and rarely necessitates additional immunosuppressant use.

Tocilizumab, a humanized monoclonal antibody directed against IL-6 receptor is FDA-approved for treatment of RA. ${ }^{24}$ A variety of cutaneous adverse events associated with tocilizumab have been described. New onset and worsening psoriasis has been rarely described in patients receiving tocilizumab. ${ }^{25-27}$ Our patient developed de novo psoriasis 3 weeks after the first infusion of tocilizumab, which worsened after the second infusion, prompting discontinuation of drug. Non-psoriatic cutaneous reactions in tocilizumab users including erythroderma, lupus-like syndrome, cutaneous vasculitis, palmoplantar pustulosis, purpuric eruptions, and atopic dermatitis have also been described. ${ }^{24,28}$ Onset of psoriasis with tocilizumab is an unexpected adverse event, as IL-6 is considered to contribute to the pathogenesis of psoriasis via Th17 cells and the resultant downstream effect on production of various cytokines (IL-17, IL-22, IL-6, TNFa). ${ }^{29,30}$ Other biologic agents such as rituximab ${ }^{31}$ and abatacept ${ }^{32}$ have also been associated with new onset or worsening psoriasis.

\section{Conclusion}

Alternative explanations for an adverse cutaneous event must be carefully investigated when confronted with a suspected new, atypical, or paradoxical adverse event. These include 


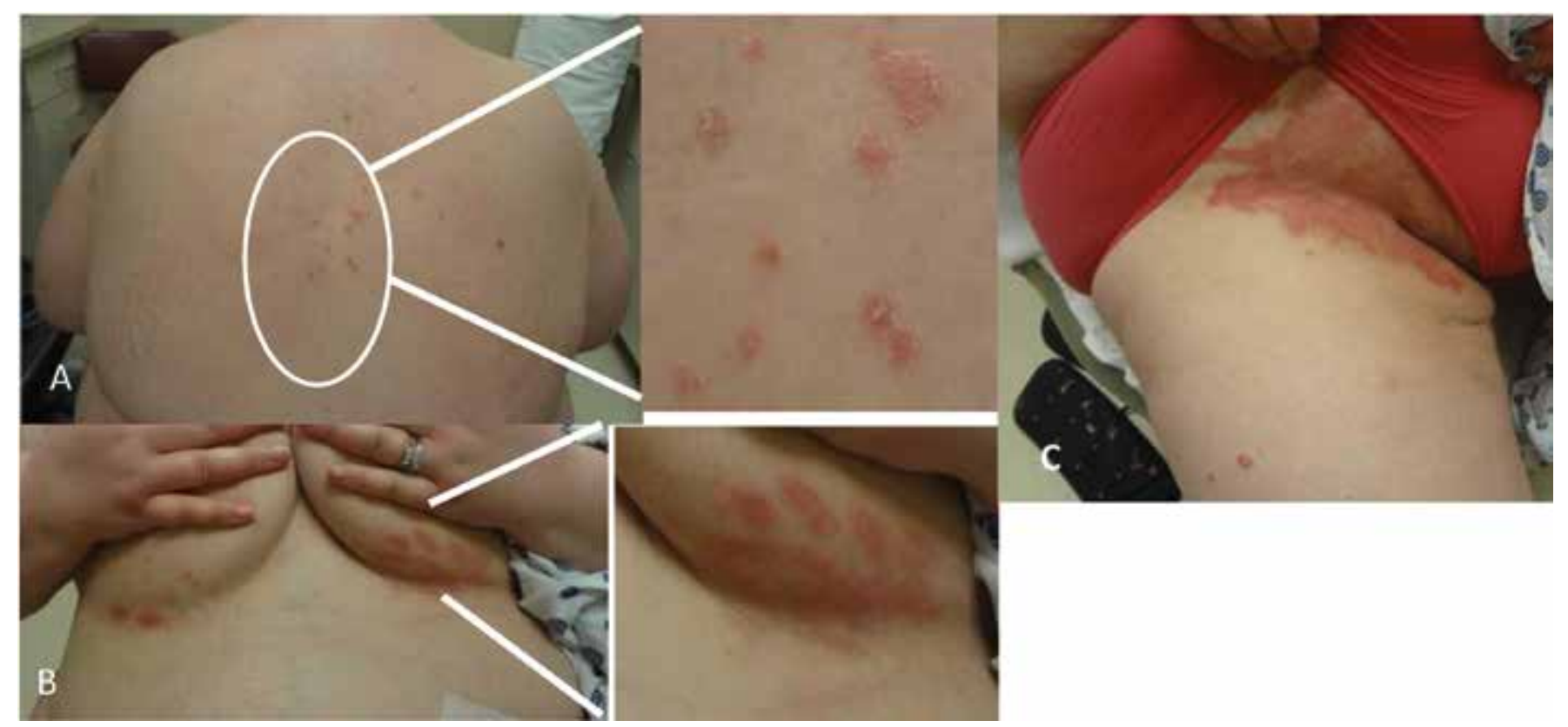

Figure 6. Red and scaly discoid lesions $(A)$ over back, $(B)$ under breasts and $(C)$ under the pannus in patient on tocilizumab.

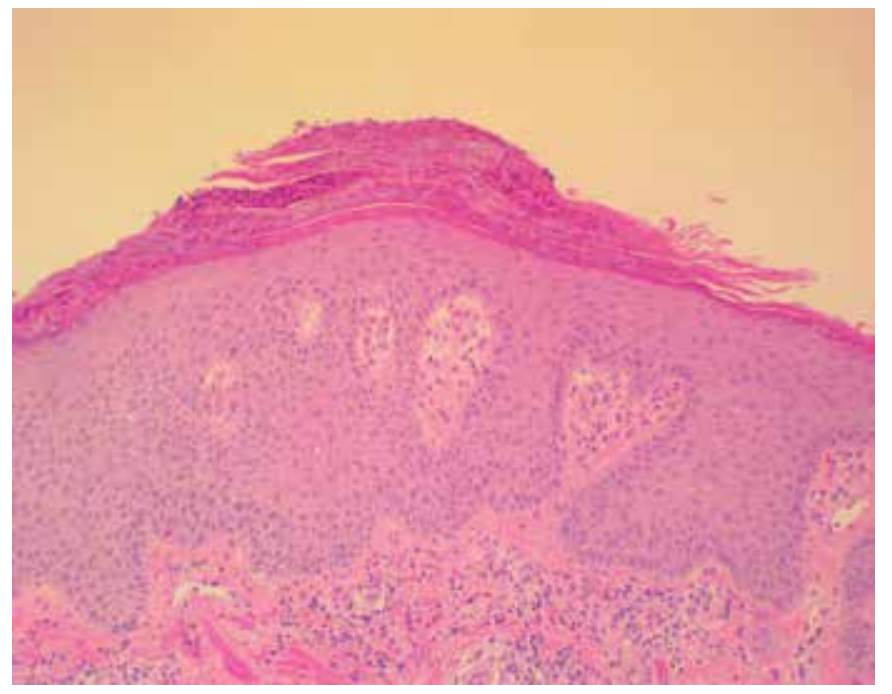

Figure 7. Sections showing psoriasiform epidermal hyperplasia with neutrophilic parakeratosis. There is suprapapillary plate thinning with prominent dermal blood vessels within dermal papillae and superficial perivascular lymphocytic infiltrate within the dermis. These findings would be in keeping with the histologic findings of psoriasis.

cutaneous manifestation of underlying disease, infusion/ injection site reactions, immune-mediated skin lesions, skin infections, eczema, and concomitant medication use. A broad range of cutaneous eruptions have been reported in patients undergoing TNF-I therapy. The pathophysiologic mechanism that triggers development of paradoxical reactions such as psoriasis is poorly understood. A close temporal relationship of the presentation and resolution of cutaneous adverse events with use and withdrawal of TNF-I therapy, respectively, is important in distinguishing a drug-induced eruption from a distinct primary process. Treatment approach is tailored to the severity of each cutaneous eruption, although discontinuation of the suspected biologic agent is a reasonable first step. Because the spectrum of adverse cutaneous events in biologic agent users continues to expand with the growing number and wider use of available biologic therapies, there is need for a greater awareness among treating physicians.

\section{References}

1. Ramos-Casals M, Roberto-Perez-Alvarez, Diaz-Lagares C, Cuadrado MJ, Khamashta MA; BIOGEAS Study Group. Autoimmune diseases induced by biological agents: a doubleedged sword? Autoimmun Rev 2010;9(3):188-193.

2. Hawryluk EB, Linskey KR, Duncan LM, Nazarian RM. Broad range of adverse cutaneous eruptions in patients on TNFalpha antagonists. J Cutan Pathol 2012;39(5):481-492.

3. Flendrie M, Vissers WHPM, Creemers MCW, de Jong EMGJ, van de Kerkhof PCM, van Riel PLCM. Dermatological conditions during TNF-alpha-blocking therapy in patients with rheumatoid arthritis: a prospective study. Arthritis Res Ther 2005;7(3):R666-R676.

4. Exarchou SA, Voulgari PV, Markatseli TE, Zioga A, Drosos AA. Immune-mediated skin lesions in patients treated with antitumour necrosis factor alpha inhibitors. Scand J Rheumatol 2009;38(5):328-331.

5. Sfikakis PP, Boletis JN, Lionaki S, Vigklis V, Fragiadaki KG, Iniotaki A, Moutsopoulos HM. Remission of proliferative lupus nephritis following B cell depletion therapy is preceded by down-regulation of the $\mathrm{T}$ cell costimulatory molecule CD40 ligand: an open-label trial. Arthritis Rheum 2005 Feb;52(2):501-13.

6. Feuerstein JD, Cheifetz AS. Miscellaneous adverse events with biologic agents (excludes infection and malignancy). Gastroenterol Clin North Am 2014;43(3):543-563.

7. Moustou AE, Matekovits A, Dessinioti C, Antoniou C, Sfikakis PP, Stratigos AJ. Cutaneous side effects of anti-tumor necrosis factor biologic therapy: A clinical review. J Am Acad Dermatol 2009;61(3):486-504.

8. Hau E, Vignon Pennamen MD, Battistella M, et al. Neutrophilic skin lesions in autoimmune connective tissue diseases: nine cases and a literature review. Medicine (Baltimore) 2014;93(29):e346. 
9. Cohen PR, Kurzrock R. Sweets syndrome revisited: a review of disease concepts. Int J Dermatol 2003;42(10):761-778.

10. Rochet NM, Chavan RN, Cappel MA, Wada DA, Gibson LE. Sweet syndrome: Clinical presentation, associations, and response to treatment in 77 patients. J Am Acad Dermatol 2013;69(4):557-564.

11. Keidel S, McColl A, Edmonds S. Sweet's syndrome after adalimumab therapy for refractory relapsing polychondritis. BMJ Case Rep 2011 Dec 21;2011. pii: bcr1020114935. doi: 10.1136/bcr.10.2011.4935.

12. Jaimes-López N, Molina V, Arroyave JE, et al. Development of pyoderma gangrenosum during therapy with infliximab. J Dermatol Case Rep 2009;3(2):20-23.

13. Brunasso AMG, Laimer M, Massone C. Paradoxical reactions to targeted biological treatments: A way to treat and trigger? Acta Derm Venereol 2010;90(2):183-185.

14. Vandevyvere K, Luyten FP, Verschueren P, Lories R, Segaert S, Westhovens R. Pyoderma gangrenosum developing during therapy with TNF-alpha antagonists in a patient with rheumatoid arthritis. Clin Rheumatol 2007;26(12):22052206.

15. Fonder MA, Cummins DL, Ehst BD, Anhalt GJ, Meyerle JH. Adalimumab therapy for recalcitrant pyoderma gangrenosum. J Burns Wounds 2006;5:e8.

16. Juillerat $P$, Christen-Zäch $S$, Troillet FX, Gallot-Lavallée $S$, Pannizzon RG, Michetti P. Infliximab for the treatment of disseminated pyoderma gangrenosum associated with ulcerative colitis. Case report and literature review. Dermatology 2007;215(3):245-251.

17. Cohen PR. Sweets syndrome - a comprehensive review of an acute febrile neutrophilic dermatosis. Orphanet J Rare Dis 2007;2(1):34.

18. Voigtländer C, Lüftl M, Schuler G, Hertl M. Infliximab (antitumor necrosis factor alpha antibody): a novel, highly effective treatment of recalcitrant subcorneal pustular dermatosis (Sneddon-Wilkinson disease). Arch Dermatol 2001;137(12):1571-1574.

19. Bonifati C, Trento E, Cordiali Fei P, Muscardin L, Amantea A, Carducci M. Early but not lasting improvement of recalcitrant subcorneal pustular dermatosis (SneddonWilkinson disease) after infliximab therapy: relationships with variations in cytokine levels in suction blister fluids. Clin Exp Dermatol 2005;30(6):662-665.

20. Marzano AV, Fanoni D, Antiga E, et al. Expression of cytokines, chemokines and other effector molecules in two prototypic autoinflammatory skin diseases, pyoderma gangrenosum and Sweets syndrome. Clin Exp Immunol 2014; 178(1):48-56.

21. Srivastava MD, Alexander F, Tuthill RJ. Immunology of cutaneous vasculitis associated with both etanercept and infliximab. Scand J Immunol 2005;61(4):329-336.

22. Mohan N, Edwards ET, Cupps TR, et al. Leukocytoclastic vasculitis associated with tumor necrosis factor-alpha blocking agents. J Rheumatol 2004;31(10):1955-1958.

23. De Miguel S, Jover JA, Vadillo C, Judez E, Loza E, FernandezGutierrez B. B cell activation in rheumatoid arthritis patients under infliximab treatment. Clin Exp Rheumatol 2003;21(6):726-732.

24. Laurent S, Le Parc JM, Clérici T, Bréban M, Mahé E. Onset of psoriasis following treatment with tocilizumab. $\mathrm{Br} \mathrm{J}$ Dermatol 2010;163(6):1364-1365.

25. Laurent S, Le Parc JM, Clérici T, Bréban M, Mahé E. Onset of psoriasis following treatment with tocilizumab. Br J Dermatol 2010;163(6):1364-1365.

26. Wendling D, Letho-Gyselinck H, Guillot X, Prati C. Psoriasis onset with tocilizumab treatment for rheumatoid arthritis. J Rheumatol 2012;39(3):657.
27. Palmou-Fontana N, Sánchez Gaviño JA, McGonagle D, García-Martinez E, Iñiguez de Onzoño Martín L. Tocilizumab-induced psoriasiform rash in rheumatoid arthritis. Dermatology 2014;228(4):311-313.

28. Ogata A, Kumanogoh A, Tanaka T. Pathological role of IL-6 in psoriatic arthritis. Arthritis 2012;2012:713618.

29. Goodman WA, Levine AD, Massari JV, Sugiyama H, McCormick TS, Cooper KD. IL-6 signaling in psoriasis prevents immune suppression by regulatory T cells. J Immunol 2009;183(5):3170-3176.

30. Lindhaus C, Tittelbach J, Elsner P. Cutaneous side effects of TNF-alpha inhibitors. J Dtsch Dermatol Ges 2017;15(3):281288.

31. Markatseli TE, Kaltsonoudis ES, Voulgari PV, Zioga A, Drosos AA. Induction of psoriatic skin lesions in a patient with rheumatoid arthritis treated with rituximab. Clin Exp Rheumatol 2009;27(6):996-998.

32. Florent A, Albert C, Giacchero D, Roux C, Euller-Ziegler L. Reactivation of cutaneous psoriasis during abatacept therapy for spondyloarthropathy. Joint Bone Spine 2010;77(6):626627.

\section{Author Affiliations}

Rahul Sehgal, MD*; Erik J. Stratman, MD†; Jonathan E. Cutlan, MD $\neq$

*Mayo Clinic Health System, Department of Rheumatology, Eau Claire, WI 54702 USA

† Marshfield Clinic Health System, Department of

Dermatology, Marshfield, WI 54449 USA

†Marshfield Clinic Health System, Department of Pathology, Marshfield, WI 54449 USA 